\title{
ON THE JOINT DISTRIBUTION OF TAX PAYMENTS AND CAPITAL INJECTIONS FOR A LÉVY RISK MODEL
}

\author{
BY
}

HANSJÖRG ALBRECHER* (LAUSANNE) AND JEVGENIJS IVANOVS (AARHUS)

Dedicated to Tomasz Rolski on the occasion of his 70th birthday

\begin{abstract}
We study the joint distribution of tax payments according to a loss-carry-forward scheme and capital injections in a Lévy risk model, and provide a transparent expression for the corresponding transform in terms of the scale function. This allows us to identify the net present value of capital injections in such a model, complementing the one for tax found in our paper [3]. We also apply the result to the situation when injections may be stopped at a constant rate, and in this case an explicit formula for the net present value of taxes and injections is given.
\end{abstract}

2010 AMS Mathematics Subject Classification: Primary: 60G51; Secondary: 60E10.

Key words and phrases: Two-sided reflection, finite buffer, dividends, net present value, scale functions.

\section{INTRODUCTION}

Following [3] we consider an insurance surplus process

$$
Y_{t}=X_{t}+L_{t}-\gamma U_{t},
$$

where $X_{t}$ is the driving spectrally-negative Lévy process started at $x \geqslant 0, \gamma U_{t}$ denotes the total tax paid at rate $\gamma \in[0,1]$ according to loss-carry-forward scheme [2], and $L_{t}$ means the total amount of capital injections required to keep the company solvent. These notions are best understood separately: without taxation the process at time $t$ is given by $X_{t}+\left(-\underline{X}_{t} \vee 0\right)$, whereas without capital injections this process is $X_{t}-\gamma\left(\bar{X}_{t}-X_{0}\right)$, where $\underline{X}_{t}=\inf _{s \leqslant t} X_{s}$ and $\bar{X}_{t}=\sup _{s \leqslant t} X_{s}$. Here and in the rest of the paper it is assumed that taxation is applied immediately, i.e.,

\footnotetext{
* Financial support by the Swiss National Science Foundation Project 200020143889 is gratefully acknowledged.
} 
from the level $X_{0}=x$. In the model with both tax and capital injections, however, the respective quantities $L_{t}$ and $U_{t}$ are closely interrelated. The rigorous definition proceeds by partitioning time into intervals and applying taxation and injection of capital locally [3].

Importantly, for $\gamma=1$ we obtain the two-sided reflection of $X_{t}$ in the interval $[0, x]$, see [5], Section XIV.3. Such a process is often used in queueing theory to model a workload process in case of a finite buffer size. In risk theory, it is common to speak in this case of dividends and capital injections or refinancing, see [8]-[10] and [13] for related work.

In this paper we provide an explicit formula for

$$
\mathbb{E}_{x}^{\gamma} e^{-\alpha U_{e_{\delta}}-\beta L_{e_{\delta}}}=\delta \int_{0}^{\infty} \mathbb{E}_{x}^{\gamma} e^{-\delta t-\alpha U_{t}-\beta L_{t}} \mathrm{~d} t
$$

the joint transform of the total tax collected (up to scaling by $\gamma$ ) and the total capital injected up to an independent exponential time $e_{\delta}$ with rate $\delta>0$, which may also be seen as a triple transform. The notation $\mathbb{E}_{x}^{\gamma}$ is used to keep the parameters $x$, the initial capital, and $\gamma$, the taxation rate, explicit. This transform may be used to study the dependence of $L_{t}$ and $U_{t}$. Moreover, the transform of the marginal $L_{e_{\delta}}$ allows us to identify the net present value of capital injections with discount rate $\delta$, i.e.,

$$
\mathbb{E}_{x}^{\gamma} \int_{0}^{\infty} e^{-\delta t} \mathrm{~d} L_{t}=\mathbb{E}_{x}^{\gamma} \int_{0}^{\infty} 1_{\left\{t<e_{\delta}\right\}} \mathrm{d} L_{t}=\mathbb{E}_{x}^{\gamma} L_{e_{\delta}},
$$

which is missing in [3], where the focus was on power identities in various models with tax. In the last equality in $(\mathbb{L}$. I) we used the fact that $L$ does not jump at $e_{\delta}$ a.s. A similar conclusion holds with respect to the net present value of tax, but this quantity can be obtained in a more direct way, cf. [3].

After some preliminaries in Section ¿, we establish the expression for the joint transform in Section [3. In Section 4 we then apply this result to obtain a transparent solution to a related problem, when the overall amount of capital injections is limited by an exponential random variable.

\section{PRELIMINARIES}

Let $\psi(s)=\log \mathbb{E} e^{s X_{1}}, s \geqslant 0$, be the Laplace exponent of the spectrally-negative Lévy process $X$. We write $\psi_{\delta}(\cdot)=\psi(\cdot)-\delta$ for the Laplace exponent of $X$ killed at an independent exponential time $e_{\delta}$, and denote the unique positive zero of $\psi_{\delta}(\cdot)$ by $\Phi_{\delta}$.

Furthermore, let $W_{\delta}(x), x \geqslant 0$, be the scale function corresponding to the killed $X$, that is, $W_{\delta}(x)$ is a continuous function characterized by its transform $\int_{0}^{\infty} e^{-s x} W_{\delta}(x) \mathrm{d} x=1 / \psi_{\delta}(s)$ for $s$ large enough. The reader is referred to [14] 
for a nice summary of the theory of scale functions. In addition, we define a socalled second scale function for any $\beta, x \geqslant 0$ by

$$
Z_{\delta, \beta}(x):=e^{\beta x}\left(1-\psi_{\delta}(\beta) \int_{0}^{x} e^{-\beta y} W_{\delta}(y) \mathrm{d} y\right),
$$

and note that its derivative in $x$,

$$
Z_{q, \beta}^{\prime}(x)=\beta Z_{q, \beta}(x)-\psi_{q}(\beta) W_{q}(x),
$$

has a simple form.

Define the following first passage times:

$$
\tau_{y}=\inf \left\{t \geqslant 0: Y_{t}>y\right\}, \quad \rho_{y}=\inf \left\{t \geqslant 0: U_{t}>y\right\},
$$

so that $\tau_{y}=\rho_{(y-x) /(1-\gamma)}$ for $y \geqslant x$ under $\mathbb{P}_{x}^{\gamma}$, which also shows that $\tau_{y}=\infty$ for $\gamma=1$. In [3] the following identities were established:

$$
\begin{array}{ll}
\mathbb{E}_{x}^{\gamma}\left(e^{-\delta \tau_{y}-\beta L_{\tau_{y}}}\right)=\left(\frac{Z_{\delta, \beta}(x)}{Z_{\delta, \beta}(y)}\right)^{1 /(1-\gamma)}, & \gamma \neq 1, \\
\mathbb{E}_{x}^{1}\left(e^{-\delta \rho_{y}-\beta L_{\rho_{y}}}\right)=\exp \left(-\frac{Z_{\delta, \beta}^{\prime}(x)}{Z_{\delta, \beta}(x)} y\right), & \gamma=1 .
\end{array}
$$

In [I]], Section 8.3, it was observed that $\left(\rho_{y}, L_{\rho_{y}}\right), y \geqslant 0$, is a bivariate Lévy process under $\mathbb{P}_{x}^{1}$ with the Laplace exponent $Z_{\delta, \beta}^{\prime}(x) / Z_{\delta, \beta}(x)$ (up to minus signs), which also leads to (2.3)).

\section{THE TRANSFORM}

The following is our main result.

THEOREM 3.1. For any $\alpha, \beta \geqslant 0$ and $x, \delta>0$ we have

$$
\mathbb{E}_{x}^{\gamma} e^{-\alpha U_{e_{\delta}}-\beta L_{e_{\delta}}}=\frac{1}{1-\gamma} \int_{x}^{\infty}\left(\frac{e^{\alpha x} Z_{\delta, \beta}(x)}{e^{\alpha y} Z_{\delta, \beta}(y)}\right)^{1 /(1-\gamma)} z_{\delta, \beta}(y) \mathrm{d} y, \quad \gamma \in[0,1),
$$

and

$$
\mathbb{E}_{x}^{1} e^{-\alpha U_{e_{\delta}}-\beta L_{e_{\delta}}}=\frac{Z_{\delta, \beta}(x)}{\alpha Z_{\delta, \beta}(x)+Z_{\delta, \beta}^{\prime}(x)} z_{\delta, \beta}(x), \quad \gamma=1
$$

where

$$
z_{\delta, \beta}(x)=Z_{\delta, 0}^{\prime}(x)-\left(Z_{\delta, 0}(x)-1\right) \frac{Z_{\delta, \beta}^{\prime}(x)}{Z_{\delta, \beta}(x)}
$$


Before giving a proof of Theorem B.1] we need to establish some auxiliary results. The first concerns the transform of $L_{e_{\delta}}$ in the simple case of no taxation: $\gamma=0$. This result may be known in the literature in some form because $L$ under $\mathbb{E}_{x}^{0}$ is the unused capacity process of a queue fed by a spectrally negative $X$, see [ $[7]$, Section 4.2, for related results. We could not find a reference though, and so a simple proof is provided.

LEMMA 3.1. We have

$$
\mathbb{E}_{x}^{0} e^{-\beta L_{e_{\delta}}}=1-Z_{\delta, 0}(x)+Z_{\delta, \beta}(x) \frac{\delta}{\Phi_{\delta}} \frac{\beta-\Phi_{\delta}}{\psi_{\delta}(\beta)} .
$$

P r o of. Using the strong Markov property of $X$, observe that

$$
\mathbb{E}_{x}^{0} e^{-\beta L_{e_{\delta}}}=\mathbb{P}_{x}\left(T_{0}>e_{\delta}\right)+\mathbb{E}_{x}\left[e^{\beta X_{T_{0}}} ; T_{0}<e_{\delta}\right] \mathbb{E}_{0}^{0} e^{-\beta L_{e_{\delta}}},
$$

where $T_{0}=\inf \left\{t \geqslant 0: X_{t}<0\right\}$, and $\mathbb{P}_{x}$ denotes the law of the free process started in $x$. Note that $L_{e_{\delta}}=-\underline{X}_{e_{\delta}}$ when the reflected process starts at zero, and the transform of this quantity is well known. The generalized Pollaczek-Khintchine formula (see [15], Theorem 4.8), or the Wiener-Hopf factorisation, states that

$$
\mathbb{E}_{0}^{0} e^{-\beta L_{e_{\delta}}}=\frac{\delta}{\Phi_{\delta}} \frac{\beta-\Phi_{\delta}}{\psi_{\delta}(\beta)} .
$$

The exit identities corresponding to $\tau_{0}^{-}$can be found in [15], Theorem 8.1(ii), and its generalization in [11], Proposition 8.1. In this way we obtain

$$
\begin{aligned}
\mathbb{E}_{x}^{0} e^{-\beta L_{e_{\delta}}}= & \left(1-Z_{\delta, 0}(x)+W_{\delta}(x) \frac{\delta}{\Phi_{\delta}}\right) \\
& +\left(Z_{\delta, \beta}(x)-W_{\delta}(x) \frac{\psi_{\delta}(\beta)}{\beta-\Phi_{\delta}}\right) \frac{\delta}{\Phi_{\delta}} \frac{\beta-\Phi_{\delta}}{\psi_{\delta}(\beta)},
\end{aligned}
$$

which readily leads to the claimed identity.

REMARK 3.1. Note that by virtue of observation (ㅍ. I), the (negative of the) first derivative of (B.2) with respect to $\beta$ at zero gives as a special case the net present value of capital injections without taxation, and after some algebra one obtains

$$
\mathbb{E}_{x}^{0} \int_{0}^{\infty} e^{-\delta t} \mathrm{~d} L_{t}=\frac{Z_{\delta, 0}(x)}{\Phi_{\delta}}-\int_{0}^{x} Z_{\delta, 0}(y) \mathrm{d} y-\frac{\psi^{\prime}(0)}{\delta},
$$

which coincides with Theorem 1 of Avram et al. [6], when using the limit relation $\lim _{x \rightarrow \infty} Z_{\delta, 0}(x) / Z_{\delta, 0}^{\prime}(x)=1 / \Phi_{\delta}$.

It has been realised in [3] that the basic reason for the power identities as in (2.2) is the memoryless property of the system for $\gamma=1$ at the times $\rho_{y}$, which also leads to certain invariance statements with respect to $\gamma$. A similar invariance statement given in the following lemma will be crucial in the present study as well. 
LEMMA 3.2. We have

$$
\mathbb{E}_{x}^{\gamma}\left[e^{-\beta L_{e_{\delta}}} ; U_{e_{\delta}}<h\right]=\mathbb{E}_{x}^{1}\left[e^{-\beta L_{e_{\delta}}} ; U_{e_{\delta}}<h\right]+o(h) \quad \text { as } h \downarrow 0 .
$$

Proof. Assume that $Y$ is sent to some cemetery state (and $U, L$ are then fixed to remain constant) at $e_{\delta}$, and consider the excursions of $Y$ from its maximum which either end up in the cemetery state or lead to the increase of $L$; here we do not need tools from Itô's excursion theory. For the special case of $\gamma=1$ observe the lack of memory property at the times when $Y$ is at its upper boundary. This lack of memory implies that there is at most one excursion as outlined above starting in the time interval $\left[0, \rho_{2 h}\right)$; here and in the following we ignore $o(h)$ probabilities. So it is left to analyse three cases for $\gamma=1$ : (i) there is no excursion as above starting in $\left[0, \rho_{h}\right)$; (ii) there is one excursion starting in $\left[0, \rho_{h}\right)$, none in $\left[\rho_{h}, \rho_{2 h}\right)$, and the excursion does not finish in the cemetery state; and (iii) this latter excursion does finish in the cemetery state. It is not difficult to see that in the cases (i) and (ii) we necessarily have $U_{e_{\delta}} \geqslant h$ for all $\gamma \in[0,1]$. In the case (iii) we must have $U_{e_{\delta}}<h$ for all $\gamma$, and moreover the differences of $L_{e_{\delta}}$ for all $\gamma$ are bounded by $h$. Thus

$$
\begin{aligned}
\mid \mathbb{E}_{x}^{\gamma}\left[e^{-\beta L_{e_{\delta}}} ; U_{e_{\delta}}<h\right]- & \mathbb{E}_{x}^{1}\left[e^{-\beta L_{e_{\delta}}} ; U_{e_{\delta}}<h\right] \mid \\
& \leqslant\left(e^{\beta h}-1\right) \mathbb{E}_{x}^{1}\left[e^{-\beta L_{e_{\delta}}} ; U_{e_{\delta}}<h\right]+o(h)=o(h),
\end{aligned}
$$

which completes the proof.

We are ready to give the proof of the main result.

P r o of of Th e o r e m 3.1. From the strong Markov property and (2.3) we obtain

$$
\begin{aligned}
\mathbb{E}_{x}^{1}\left[e^{-\beta L_{e_{\delta}}} ; U_{e_{\delta}}<h\right] & =\mathbb{E}_{x}^{1} e^{-\beta L_{e_{\delta}}}-\mathbb{E}_{x}^{1}\left[e^{-\beta L_{\rho_{h}}} ; \rho_{h}<e_{\delta}\right] \mathbb{E}_{x}^{1} e^{-\beta L_{e_{\delta}}} \\
& =\mathbb{E}_{x}^{1} e^{-\beta L_{e_{\delta}}} \frac{Z_{\delta, \beta}^{\prime}(x)}{Z_{\delta, \beta}(x)} h+o(h) .
\end{aligned}
$$

Denoting the latter expression by $z_{\delta, \beta}(x) h+o(h)$, we use Lemma 3.2 to find that

$$
\mathbb{E}_{x}^{\gamma}\left[e^{-\beta L_{e_{\delta}}} ; U_{e_{\delta}}<h\right]=z_{\delta, \beta}(x) h+o(h) \quad \text { for all } \gamma .
$$

Letting $A_{\alpha, \beta}^{\gamma, \delta}(x)=\mathbb{E}_{x}^{\gamma} e^{-\alpha U_{e_{\delta}}-\beta L_{e}}$, we condition on $U_{e_{\delta}} \in \mathrm{d} y$ and use the strong Markov property at $\rho_{y}$ to obtain

$$
A_{\alpha, \beta}^{\gamma, \delta}(x)=\int_{0}^{\infty} e^{-\alpha y} \mathbb{E}_{x}^{\gamma}\left(e^{-\beta L_{\rho_{y}}} ; \rho_{y}<e_{\delta}\right) z_{\delta, \beta}(x+(1-\gamma) y) \mathrm{d} y
$$

because at the time $\rho_{y}$ the process is at the level $x+(1-\gamma) y$. More precisely, we sum up over the events $U_{e_{\delta}} \in\left[y_{i}, y_{i+1}\right)$ for some partition of $[0, C)$, and then let 
the mesh of the partition go to zero followed by $C \rightarrow \infty$, which results in the above Riemann integral; one may also check that the integrand is a continuous function of $y$ and hence Riemann integrable.

Now for $\gamma \neq 1$ the identity (2.2) implies that

$$
A_{\alpha, \beta}^{\gamma, \delta}(x)=\int_{0}^{\infty} e^{-\alpha y}\left(\frac{Z_{\delta, \beta}(x)}{Z_{\delta, \beta}(x+(1-\gamma) y)}\right)^{1 /(1-\gamma)} z_{\delta, \beta}(x+(1-\gamma) y) \mathrm{d} y
$$

because $\rho_{y}=T_{x+(1-\gamma) y}$. Change of variable leads to the result for $\gamma \neq 1$ up to identification of $z_{\delta, \beta}(x)$. Similar manipulations using (2.3) yield the result also for $\gamma=1$.

It is left to determine $z_{\delta, \beta}(x)$. Note that

$$
A_{0, \beta}^{0, \delta}(x)=\int_{x}^{\infty} \frac{Z_{\delta, \beta}(x)}{Z_{\delta, \beta}(y)} z_{\delta, \beta}(y) \mathrm{d} y,
$$

which upon division by $Z_{\delta, \beta}(x)$ and differentiation at $x$ reduces to

$$
z_{\delta, \beta}(x)=-Z_{\delta, \beta}(x)\left(A_{0, \beta}^{0, \delta}(x) / Z_{\delta, \beta}(x)\right)^{\prime} .
$$

Using Lemma B.] we find that $z_{\delta, \beta}(x)=Z_{\delta, \beta}(x)\left(\left(Z_{\delta, 0}(x)-1\right) / Z_{\delta, \beta}(x)\right)^{\prime}$, which readily leads to (B.T).

Let us note that Theorem 3.$]$ identifying the transform $\mathbb{E}_{x}^{1} e^{-\alpha U_{e_{\delta}}-\beta L_{e_{\delta}}}$ complements the results in the literature concerning the distribution of $Y_{e_{\delta}}$ under $\mathbb{E}_{x}^{1}$, which essentially is the $\delta$-potential measure of the process $Y_{t}$. In fact, the latter distribution is known even in a more general setting of a Markov additive process and an arbitrary initial value ([112], Theorem 2). Furthermore, there is a simple sample path identity relating capital injections in the original model and dividends in the time-reversed counterpart, see [4].

Finally, it is rather straightforward to extend the result of Theorem 3.7 to the case of delayed taxation, i.e., when taxation is applied from some level $y \geqslant x$ onward. The main ingredients are given by (2.2) for $\gamma=0$ and Lemma 3.1 .

\section{AN UPPER LIMIT FOR INJECTIONS}

Let us reconsider the model from [3] where the investor sets a random upper limit for the overall injections, and this limit is exponentially distributed with rate $\theta \geqslant 0$. One interpretation of this is in terms of lack of memory of the investor, namely that irrespective of the previous investments, injections are stopped with a constant probability at each infinitesimal amount of necessary payment (e.g., because interest in this corporation is lost, or due to exogeneous factors that are independent of the risk process). Once the payments are stopped, the process is 
declared ruined (since at that point in time the surplus is necessarily negative), and no further injections or tax payments take place. Note that for $\theta=0$ we recover our original model (without a limit for injections), and for $\theta=\infty$ we recover the model without injections at all (for the classical ruin and tax model, cf. [U]]). Apart from the above interpretation, a further motivation for considering this model variant is that it leads to a simple formula.

Denote the net present value of capital injections and tax in this setup by $V_{I}^{\gamma}(x, \delta, \theta)$ and $V_{T}^{\gamma}(x, \delta, \theta)$, respectively. Let $M \in[0, \infty]$ be the (random) limit on the capital that the investor agrees to spend. Then, by definition, the injections will be stopped as soon as $L_{t}>M$, which will happen during an injection payment. The expected discounted injections are then given by

$$
\begin{aligned}
\mathbb{E}_{x}^{\gamma}\left(\int_{0}^{\infty} e^{-\delta t} 1_{\left\{L_{t}<M\right\}} \mathrm{d} L_{t}+\right. & \left.e^{-\delta \tau}\left(M-L_{\tau-}\right)\right) \\
& =\mathbb{E}_{x}^{\gamma}\left(\int_{0}^{e_{\delta}} 1_{\left\{L_{t}<M\right\}} \mathrm{d} L_{t}+1_{\left\{\tau<e_{\delta}\right\}}\left(M-L_{\tau-}\right)\right),
\end{aligned}
$$

where $\tau$ is the time when $L_{t}$ exceeds $M$ (and ruin is declared). By considering the event $L_{e_{\delta}}<M$ and its complement we see that the above formula simplifies to

$$
\mathbb{E}_{x}^{\gamma}\left(L_{e_{\delta}} \wedge M\right)
$$

because $\tau \neq e_{\delta}$ and $L$ does not jump at $e_{\delta}$ a.s. Thus we arrive at the formula

$$
V_{I}^{\gamma}(x, \delta, \theta)=\mathbb{E}_{x}^{\gamma}\left(L_{e_{\delta}} \wedge e_{\theta}\right)=\frac{1}{\theta}\left(1-\mathbb{E}_{x}^{\gamma} e^{-\theta L_{e_{\delta}}}\right),
$$

and so Theorem 3.0 readily applies.

Instead of the interpretation as tax, the payments $\gamma U_{t}$ may also be considered as particular proportional profit participation scheme for the investor, who in turn provides the capital injections in case of negative surplus. Then the balance between received and provided payments is of particular interest (in the interpretation of tax, one may also view the injections as bail-out strategies of the state in case the company has strategic or economic importance for the society, and the stopping of injections may then, e.g., be due to political circumstances). The following result identifies the net present value of profit

$$
V^{\gamma}(x)=V_{T}^{\gamma}(x, \delta, \theta)-\eta V_{I}^{\gamma}(x, \delta, \theta)
$$

in this situation where $\eta>0$ is some penalty factor.

THEOREM 4.1. We have

$$
\begin{aligned}
V^{\gamma}(x) & =\frac{1}{1-\gamma} \int_{x}^{\infty}\left(\frac{Z_{\delta, \theta}(x)}{Z_{\delta, \theta}(y)}\right)^{1 /(1-\gamma)}\left(\gamma+\frac{\eta}{\theta} z_{\delta, \theta}(y)\right) \mathrm{d} y-\frac{\eta}{\theta}, & & \gamma \in[0,1), \\
V^{1}(x) & =\frac{Z_{\delta, \theta}(x)}{Z_{\delta, \theta}^{\prime}(x)}\left(1+\frac{\eta}{\theta} z_{\delta, \theta}(x)\right)-\frac{\eta}{\theta}, & \gamma & =1 .
\end{aligned}
$$


Moreover, for $\eta \geqslant 1$ and any $\gamma \in[0,1]$ the function $V_{\gamma}(x)$ is increasing in $x>0$, and

$$
\lim _{x \rightarrow \infty} V^{\gamma}(x)=\gamma / \Phi_{\delta}
$$

Pro of. From [3] or directly from ([2.2) and (2.3) we find for $0 \leqslant \gamma<1$ that

$$
V_{T}^{\gamma}(x, \delta, \theta)=\frac{\gamma}{1-\gamma} \int_{x}^{\infty}\left(\frac{Z_{\delta, \theta}(x)}{Z_{\delta, \theta}(y)}\right)^{1 /(1-\gamma)} \mathrm{d} y
$$

and similarly $V_{T}^{1}(x, \delta, \theta)=Z_{\delta, \theta}(x) / Z_{\delta, \theta}^{\prime}(x)$. Using (4.]) and Theorem [.], we readily establish the two formulas for $V^{\gamma}(x)$.

Finally, a careful comparison of the sample paths shows that $V^{\gamma}(x)$ must be increasing when $\eta \geqslant 1$, and the limit follows from a simple calculation:

$$
V^{\gamma}(\infty)=\gamma \mathbb{E} \int_{0}^{\infty} e^{-\delta \tau_{y}^{+}} \mathrm{d} y=\gamma \mathbb{E} \int_{0}^{\infty} e^{-\Phi_{\delta} y} \mathrm{~d} y=\frac{\gamma}{\Phi_{\delta}},
$$

where $\tau_{y}^{+}$under $\mathbb{E}$ is the first passage time over the level $y$ by the free process $X$.

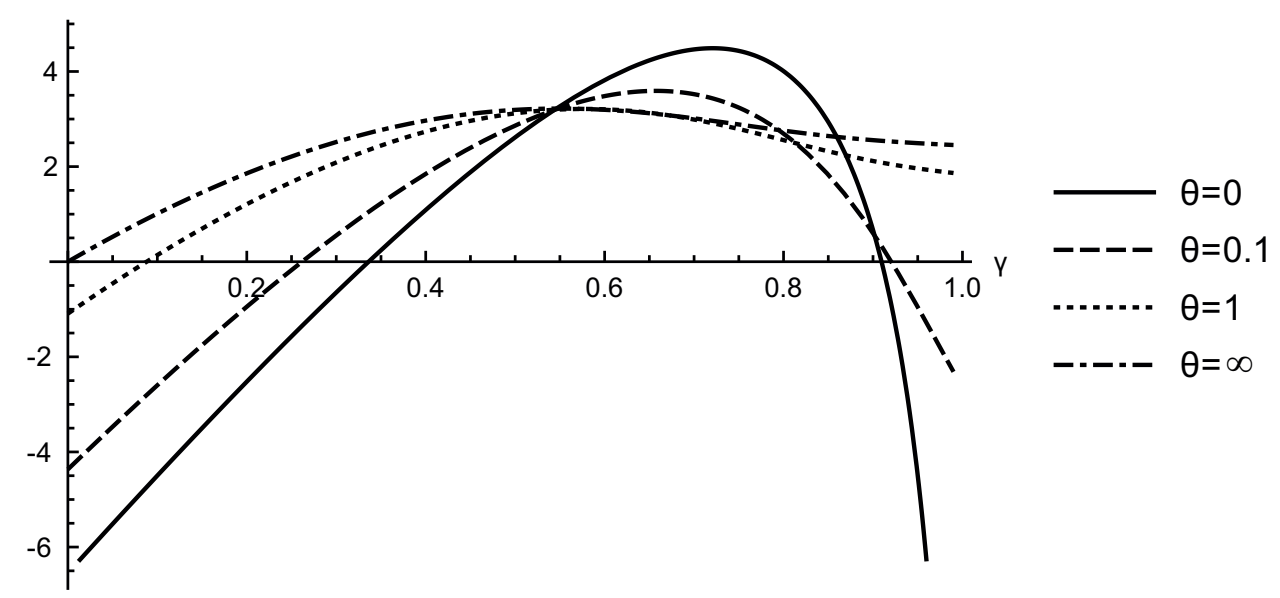

Figure 1. Net present value of profit as a function of $\gamma \in[0,1]$ for $\theta \in\{0,0.1,1, \infty\}$.

We finish with a simple numerical illustration of the result in Theorem 4.J. Let $X(t)$ be a compound Poisson process with drift (which is the classical CramérLundberg process in risk theory) with premium intensity 1.2 , claim arrival rate 1 , and exponentially distributed claim sizes of rate 1, leading to safety loading of $20 \%$. Additionally, the discount rate is set to $\delta=0.01$, and $\eta=2$, implying that injections are twice more expensive than premiums.

Figure $\square$ depicts $V^{\gamma}(1)$ as a function of the tax rate $\gamma \in[0,1]$ for various values of $\theta$. One observes that for a too small fixed $\gamma$, despite a positive drift of the process, it is preferable not to inject capital which may outweigh the small total 
gain. Also, for too high values of $\gamma$, one expects too many necessary injections to make an unlimited continuation favourable, but this depends on the choice of penalty factor $\eta$ and discount rate $\delta$. Finally, the explicit formula above allows us to look for an optimal combination of $\gamma$ and $\theta$, given that both parameters can be controlled by the investor.

\section{REFERENCES}

[1] H. Albrecher, S. Borst, O. Boxma, and J. Resing, The tax identity in risk theory - a simple proof and an extension, Insurance Math. Econom. 44 (2) (2009), pp. 304-306.

[2] H. Albrecher and C. Hipp, Lundberg's risk process with tax, Bl. DGVFM 28 (1) (2007), pp. 13-28.

[3] H. Albrecher and J. Ivanovs, Power identities for Lévy risk models under taxation and capital injections, Stoch. Syst. 4 (1) (2014), pp. 157-172.

[4] H. Albrecher and J. Ivanovs, Linking dividends and capital injections - a probabilistic approach, Scand. Actuar. J. (to appear).

[5] S. Asmussen, Applied Probability and Queues, second edition, Stoch. Model. Appl. Probab., Vol. 51, Springer, New York 2003.

[6] F. Avram, Z. Palmowski, and M. Pistorius, On the optimal dividend problem for a spectrally negative Lévy process, Ann. Appl. Probab. 17 (1) (2007), pp. 156-180.

[7] K. Dębicki and M. Mandjes, Queues and Lévy Fluctuation Theory, Universitext, Springer, Cham 2015.

[8] D. Dickson and H. R. Waters, Some optimal dividends problems, Astin Bull. 34 (1) (2004), pp. 49-74.

[9] H. U. Gerber and E. S. Shiu, Geometric Brownian motion models for assets and liabilities: From pension funding to optimal dividends, N. Am. Actuar. J. 7 (3) (2003), pp. 37-51.

[10] J. Hugonnier and E. Morellec, Bank capital, liquid reserves, and insolvency risk, J. Financ. Econ. 125 (2) (2017), pp. 266-285.

[11] J. Ivanovs, One-Sided Markov Additive Processes and Related Exit Problems, PhD dissertation, University of Amsterdam, Uitgeverij BOXPress, Oisterwijk 2011.

[12] J. Ivanovs, Potential measures of one-sided Markov additive processes with reflecting and terminating barriers, J. Appl. Probab. 51 (4) (2014), pp. 1154-1170.

[13] N. Kulenko and H. Schmidli, Optimal dividend strategies in a Cramér-Lundberg model with capital injections, Insurance Math. Econom. 43 (2) (2008), pp. 270-278.

[14] A. Kuznetsov, A. E. Kyprianou, and V. Rivero, The theory of scale functions for spectrally negative Lévy processes, in: Lévy Matters II, Springer, 2013, pp. 97-186.

[15] A. E. Kyprianou, Introductory Lectures on Fluctuations of Lévy Processes with Applications, Universitext, Springer, Berlin 2006.

Hansjörg Albrecher

University of Lausanne and Swiss Finance Institute

Quartier UNIL-Dorigny

Bâtiment Extranef

1015 Lausanne, Switzerland

E-mail: hansjoerg.albrecher@unil.ch
Jevgenijs Ivanovs

Aarhus University

Ny Munkegade 118

DK-8000 Aarhus C, Denmark

E-mail: jevgenijs.ivanovs@math.au.dk

Received on 14.12.2016;

revised version on 22.2 .2017 
Probability and Mathematical Statistics 37, 2017, z. 2 (C) for this edition by CNS 